\title{
Degree correlation effect of bipartite network on personalized recommendation
}

\author{
Jian-Guo Liu ${ }^{\mathrm{a}, \mathrm{b}, \mathrm{c}}$, Tao Zhou ${ }^{\mathrm{a}, \mathrm{b}, \mathrm{c}}$, Zhao-Guo Xuan ${ }^{\mathrm{d}}$, \\ Hong-An Che ${ }^{\text {a }}$, Bing-Hong Wang ${ }^{\text {a,b }}$, Yi-Cheng Zhang a,b,c \\ ${ }^{a}$ Research Center of Complex Systems Science, University of Shanghai for Science \\ and Technology, Shanghai 200093, P R China \\ ${ }^{\mathrm{b}}$ Department of Modern Physics, University of Science and Technology of China, \\ Hefei 230026, P R China \\ ${ }^{\mathrm{c}}$ Department of Physics, University of Fribourg, Fribourg CH-1700, Switzerland \\ ${ }^{\mathrm{d}}$ Institute of Systems Engineering, Dalian University of Technology, Dalian \\ 116024, P R China
}

\begin{abstract}
In this paper, by introducing a new user similarity index base on the diffusion process, we propose a modified collaborative filtering (MCF) algorithm, which has remarkably higher accuracy than the standard collaborative filtering. In the proposed algorithm, the degree correlation between users and objects is taken into account and embedded into the similarity index by a tunable parameter. The numerical simulation on a benchmark data set shows that the algorithmic accuracy of the MCF, measured by the average ranking score, is further improved by $18.19 \%$ in the optimal case. In addition, two significant criteria of algorithmic performance, diversity and popularity, are also taken into account. Numerical results show that the presented algorithm can provide more diverse and less popular recommendatoins, for example, when the recommendation list contains 10 objects, the diversity, measured by the hamming distance, is improved by $21.90 \%$.
\end{abstract}

Key words: Recommender systems, Collaborative filtering, Bipartite networks. PACS: 89.20.Hh, 89.75.Hc, 05.70.Ln

Email address: liujg004@ustc.edu.cn (Jian-Guo Liu). 


\section{Introduction}

With the expansion of Internet [1] and widely applications of Web 2.0, how to efficiently help people obtain information that they truly need is a challenging task nowadays [2]. Recommender systems have become an effective tool to address the information overload problem by predicting the user's interests and habits based on their historical selections or collections, which have been used to recommend books and CDs at Amazon.com, movies at Netflix.com, and news at Versifi Technologies (formerly AdaptiveInfo.com) [3]. Motivated by the practical significance to the e-commerce and society, study of recommender systems has caught increasing attentions and become an essential issue in Internet applications such as e-commerce systems and digital library systems [4]. A personalized recommender system includes three parts: data collection, model analysis and the recommendation algorithm, among which the algorithm is the core part. Various kinds of algorithms have been proposed thus far, including collaborative filtering approaches $[5,6,7,8,9]$, content-based analyses $[10,11]$, hybrid algorithms $[12,13,14]$, and so on. For a review of current progress, see Refs. [3,15] and the references therein.

One of the most successful recommendation algorithms, called collaborative filtering $(\mathrm{CF})$, has been developed and extensively investigated over the past decade $[5,6,16]$. When predicting the potential interests of a given user, $\mathrm{CF}$ algorithm firstly identifies a set of similar users from the past records and then makes predictions based on the weighted combination of those similar users' opinions. Despite its wide applications, CF algorithm suffers from several major limitations including system scalability and accuracy [17]. Therefore, the current CF algorithms still require further improvements to make recommendations more effective. Recently, some physical dynamics, including mass diffusion [18,19] and heat conduction [20], have found their applications in personalized recommendations. Liu et al. [7] introduced the mass diffusion process to compute the user similarity of $\mathrm{CF}$, and found that the modified algorithm has remarkably higher accuracy than the standard CF. In this method, all of the objects and users with far different degrees have been treated equally, in other words, the degree correlations between objects and users are neglected. For example, suppose a user with small degree has collected a small-degree object, the edge connecting them represents a very special taste of the user, while the information contained in the edges connecting an active user and a popular object is less meaningful. Therefore, we argue that the user similarity index could be improved by considering the degree correlation of the user-object bipartite network. The numerical results show that the improved index that depresses the influence of mainstream preferences can provide more accurate and more diverse recommendations. 


\section{Method}

Suppose there are $m$ objects and $n$ users in a recommender system. Denote the object set as $O=\left\{o_{1}, o_{2}, \cdots, o_{m}\right\}$ and the user set as $U=\left\{u_{1}, u_{2}, \cdots\right.$, $\left.u_{n}\right\}$, a recommender system can be fully described by an adjacent matrix $A=\left\{a_{i j}\right\} \in R^{m, n}$, where $a_{i j}=1$ if $o_{i}$ is collected by $u_{j}$, and $a_{i j}=0$ otherwise. In the standard $\mathrm{CF}$, the user or object similarities are calculated firstly, then the predictions are computed accordingly. If $u_{i}$ has not yet collected $o_{j}$ (i.e., $\left.a_{j i}=0\right)$, the predicted score, $v_{i j}$, is given as

$$
v_{i j}=\frac{\sum_{l=1}^{n} s_{l i} a_{j l}}{\sum_{l=1}^{n} s_{l i}},
$$

where $s_{l i}$ is the similarity between user $u_{l}$ and $u_{i}$. The widest used similarity indices are the Sorenson Index [?] and the Salton Index [?], however, they only rely on the users' degrees and the number of common collected objects, without consideration of the influence of degree correlation between users and objects. Inspired by the mass diffusion process proposed by Zhou et al. [19], Liu et al. [7] proposed a modified CF to improve the algorithmic accuracy by using the mass diffusion process to compute the user similarities, and they found that the diversity of recommendations is also enhanced. Although this algorithm has improved the standard CF, however, the degree correlation between users and objects has not been considered, thus every edge has the same contribution to the diffusion process. If both of $u_{i}$ and $u_{j}$ have selected an object $o_{l}$, they probably have similar tastes or interests. Provided the degree of $o_{l}$ is very large (object $o_{l}$ is very popular), this taste (the favor for $o_{l}$ ) is ordinary and it does not mean $u_{i}$ and $u_{j}$ are very similar. Therefore, its contribution to $s_{i j}$ should be weaken. On the other hand, provided that a user $u_{i}$ with small degree has collected an unpopular object $o_{l}$ (the degree of $o_{l}$ is very small), this taste should be very special, the contribution of the edge connecting $u_{i}$ and $o_{l}$ should be enlarged. It is not very meaningful if a user with large degree has selected a popular object, while if an unpopular object is selected by a small-degree user, this edge would contain rich information on personalized preference. Accordingly, the contribution of the edge connecting $u_{i}$ and $o_{l}$ should be negatively correlated with $k\left(u_{i}\right) k\left(o_{l}\right)$. We assume a certain amount of resource (e.g. recommendation power) is associated with each user, and the weight $s_{i j}$ represents the proportion of the resource $u_{j}$ would like to distribute to $u_{i}$. Following a network-based resource-allocation process where each user distributes his/her initial resource to all the objects he/she has collected, and then each object sends back what it has received to all the users who collected it, considering the correlation between users and objects, the weight $s_{i j}$ (the 
fraction of initial resource $u_{j}$ eventually gives to $u_{i}$ ) can be expressed as

$$
s_{i j}=\frac{1}{k\left(u_{j}\right)} \sum_{l=1}^{m} \frac{a_{l i}\left(k_{u_{j}} k_{o_{l}}\right)^{\lambda} \cdot a_{l j}\left(k_{u_{i}} k_{o_{l}}\right)^{\lambda}}{k\left(o_{l}\right)}
$$

where $\lambda$ is a tunable parameter controlling the effect of degree correlation. Based on the above definition, given a target user $u_{i}$, the algorithm is given as following: (i) Calculating the user similarity matrix $\left\{s_{i j}\right\} \in R^{n, n}$ based on the diffusion process, as shown in Eq. (2); (ii) For each user $u_{i}$, according to Eq. (1), calculating the predicted scores for his/her uncollected objects; (iii) Sorting the uncollected objects in descending order of the predicted scores, and those objects in the top will be recommended.

\section{Algorithmic performance metrics}

To test a recommendation method on a dataset we randomly remove $10 \%$ of the links as the probe set and apply the algorithm to the remainder (training set) to produce a recommendation list for each user. We then employ three different metrics, one to measure accuracy in recovery of deleted links and two to measure recommendation popularity and diversity.

\subsection{Average ranking score}

An accurate method will put preferable objects in higher places. The average ranking score is adopted to measure the accuracy, which is defined as follows. For an arbitrary user $u_{i}$, if the entry $u_{i}-o_{j}$ is in the probe set (according to the training set, $o_{j}$ is an uncollected object for $u_{i}$ ), we measure the position of $o_{j}$ in the ordered list. For example, if there are $L_{i}=10$ uncollected objects for $u_{i}$, and $o_{j}$ is the $3 r d$ from the top, we say the position of $o_{j}$ is $3 / 10$, denoted by $r_{i j}=0.3$. Since the probe entries are actually collected by users, a good algorithm is expected to give high recommendations to them, leading to small $r_{i j}$. Therefore, the mean value of the position, $\langle r\rangle$, averaged over all the entries in the probe, can be used to evaluate the algorithmic accuracy: the smaller the average ranking score, the higher the algorithmic accuracy, and vice verse.

\subsection{Popularity and diversity}

Besides accuracy, the average degree of all recommended objects, $\langle k\rangle$, and the

mean value of Hamming distance, $S$, are taken into account to measure the 


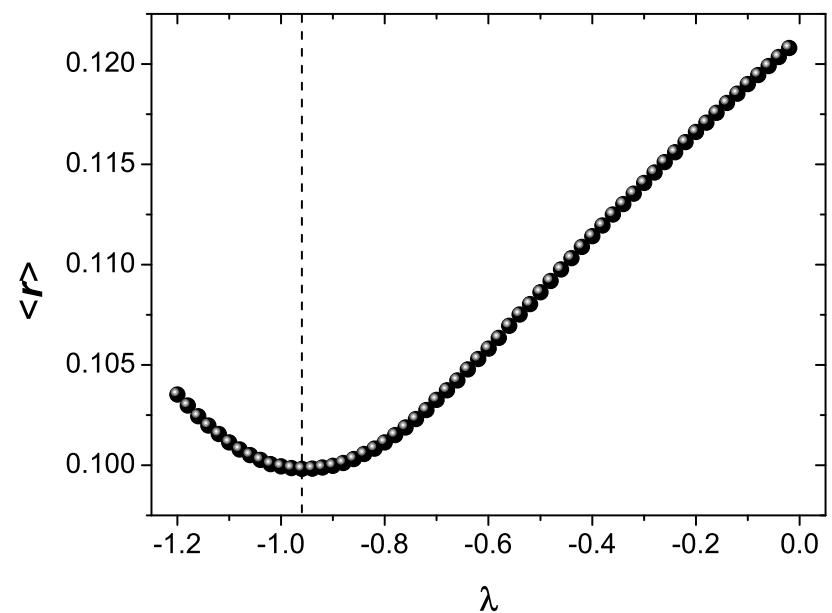

Fig. 1. The average ranking score $\langle r\rangle$ vs. $\lambda$ for the algorithm. The optimal $\lambda_{\text {opt }}$, corresponding to the minimal $\langle r\rangle=0.0998$, is $\lambda_{\text {opt }}=-0.96$. When $\lambda=0$, the algorithm degenerates to the accuracy of the $\mathrm{CF}$ based on the diffusion process. All the data points are averaged over ten independent runs with different data-set divisions.

algorithmic popularity and diversity [21]. The smaller average degree, corresponding to the less popular objects, are preferred since those lower-degree objects are hard to be found by users themselves. For example, suppose there are 10 perfect movies not yet known for user $u_{i}, 7$ of which are widely popular, while the other three fit a certain specific taste of $u_{i}$. An algorithm recommending the 7 popular movies is very nice for $u_{i}$, but he may feel even better about a recommendation list containing those two unpopular movies. In addition, the personalized recommendation algorithm should present different recommendations to different users according to their tastes and habits. The diversity can be quantified by the average Hamming distance, $S=\left\langle H_{i j}\right\rangle$, where $H_{i j}=1-Q_{i j} / L, L$ is the length of recommendation list and $Q_{i j}$ is the overlapped number of objects in $u_{i}$ and $u_{j}$ 's recommendation lists. The largest $S=1$ indicates the recommendations to all of the users are totally different, in other words, the system has highest diversity. While the smallest $S=0$ means that the recommendations for different users are exactly the same.

\section{Numerical results}

A benchmark dataset, namely MovieLens, is used to test the above algorithm, which consists of 1682 movies (objects) and 943 users. The users vote movies by discrete ratings from one to five. We applied a coarse-graining method: A movie is set to be collected by a user only if the given rating is larger than 2. The original data contains $10^{5}$ ratings, $85.25 \%$ of which are larger than 2 , 


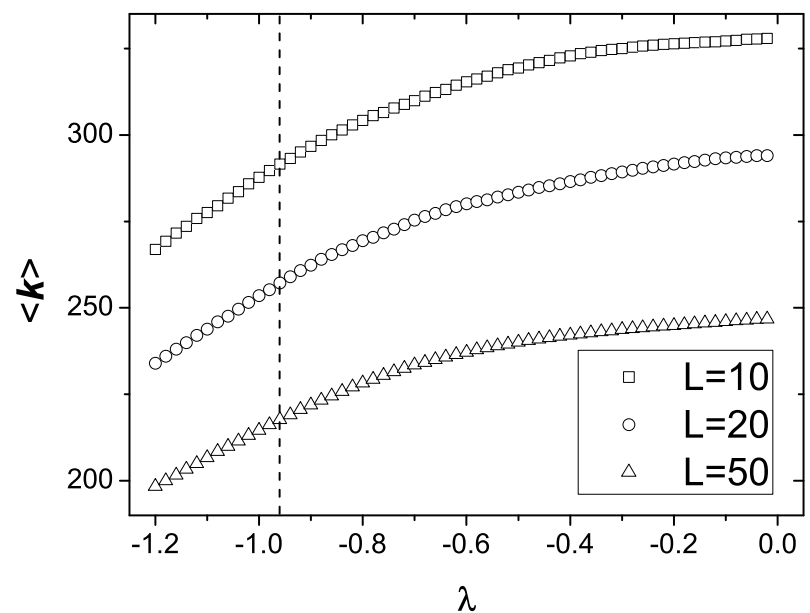

Fig. 2. Average degrees of recommended objects, $\langle k\rangle$ vs. $\lambda$. Squares, circles and triangles represent lengths $L=10,20$ and 50, respectively. All the data points are averaged over ten independent runs with different data-set divisions.

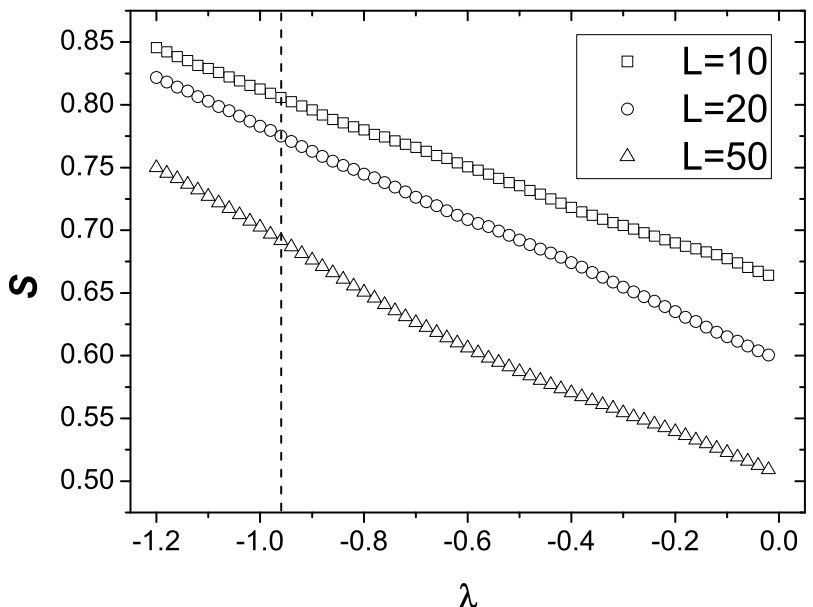

Fig. 3. The diversity $S$ vs. $\lambda$. Squares, circles and triangles represent the lengths $L=10,20$ and 50, respectively. All the data points are averaged over ten independent runs with different data-set divisions.

that is, the user-object (user-movie) bipartite network after the coarse gaining contains 85250 edges.

Figure 1 reports the algorithmic accuracy as a function of $\lambda$. The curve has a clear minimum around $\lambda=-0.96$, which strongly support the above discussion that to depress the influence of the users or objects with large degrees could enhance the accuracy. Compared with the routine case $(\lambda=0)$, the average ranking score can be reduced by $18.19 \%$ at the optimal case, which indeed a great improvement. Figure 2 reports the average degree of all recom- 
mended movies as a function of $\lambda$. When $\lambda<0$ the average object degree is positively correlated with $\lambda$, thus to depress the influence of edges connecting active users and popular objects gives more opportunity to the unpopular objects, which is consistent with our expectation. Figure 3 exhibits a negative correlation between $S$ and $\lambda$, indicating that to depress the influence of the edges connecting active users and popular objects makes the recommendations more personalized. When $L=10$, the diversity $S$ is increased from 0.661 (corresponding to the case when $\lambda=0$ ) to 0.806 (corresponding to the optimal case $\lambda=-0.96$ ), improved by $21.90 \%$.

\section{Conclusions and discussions}

In this paper, a modified collaborative filtering algorithm is presented to improve the algorithmic accuracy by depressing the influence of edges connecting active users and popular objects. The algorithmic accuracy, measured by the average ranking score, can be improved by $18.19 \%$. Beside accuracy, two significant criteria of algorithmic performance, popularity and diversity, are also taken into account. A good recommendation algorithm should not only has higher accuracy, but also help the users uncovering the hidden information, corresponding to those objects with low degrees. Therefore, the average object degree is a meaningful measurment for a recommendation algorithm. In addition, a personalized recommender system should provide each user personalized recommendations according to his/her interests and habits, therefore, the diversity of recommendations plays a crucial role to quantify the personalization. The numerical results show that the presented algorithm outperforms the standard CF in all three criteria, accuracy, popularity and diversity.

Since the power computation takes much more time than multiplication, this algorithm would take longer time to get the user similarities. Throughout the numerical simulation results, we could find that the optimal $\lambda_{\text {opt }}$ is close to -1 . When $\lambda=-1$, the corresponding $\langle r\rangle=0.0995$, which is also improved $18.07 \%$, and the average object degree and diversity are getting even better, where the diversity $S$ has been improved by $23.40 \%$. Therefore, in real application, the parameter could be set as -1 , which ensures that the algorithmic complexity is as same as a parameter free $\mathrm{CF}$.

How to automatically find out relevant information for diverse users is a longstanding challenge in the modern information science, the presented algorithm also could be used to find the relevant reviewers for the scientific papers or funding applications [22,23], and the link prediction in social and biological networks [24]. We believe the current work can enlighten readers in this promising direction. 
We acknowledge GroupLens Research Group for providing us the data set. This work is partially supported by SBF (Switzerland) project C05.0148 (Physics of Risk), the National Natural Science Foundation of China (Grant Nos. 10635040 and 60744003), the Swiss National Science Foundation (project 205120-113842), the Specialized Research Fund for the Doctoral Program of Higher Education of China.(Grant No. 20060358065), and by the Research Fund of the Education Department of Liaoning of China (20060140).

\section{References}

[1] G.-Q. Zhang, G.-Q. Zhang, Q.-F. Yang, S.-Q. Cheng, T. Zhou, New Journal of Physics 10 (2008) 12307.

[2] P. Resnick, H. R. Varian, Commun. ACM 40 (1997) 56.

[3] G. Adomavicius, A. Tuzhilin, IEEE Trans. Know. \& Data Eng. 17 (2005) 734.

[4] J. B. Schafer, J. A. Konstan, J. Riedl, Data Min. \& Knowl. Disc. 5 (2001) 115.

[5] J. L. Herlocker, J. A. Konstan, K. Terveen, J. Riedl, ACM Trans. Inform. Syst. $22(2004) 5$.

[6] J. A. Konstan, B. N. Miller, D. Maltz, J. L. Herlocker, L. R. Gordon, J. Riedl, Commun. ACM 40 (1997) 77.

[7] J.-G. Liu, B.-H. Wang, Q. Guo, Int. J. Mod. Phys. C 20 (2009) 285.

[8] J.-G. Liu, T. Zhou, B.-H. Wang, Y.-C. Zhang, arXiv:0808.3726.

[9] R.-R. Liu, C.-X. Jia, T. Zhou, D.Sun, B.-H. Wang, Physica A 388 (2009) 462.

[10] M. Balabanović, Y. Shoham, Commun. ACM 40 (1997) 66.

[11] M. J. Pazzani, Artif. Intell. Rev. 13 (1999) 393.

[12] M. Pazzani, D. Billsus, Machine Learning 27 (1997) 313.

[13] C. Basu, H. Hirsh, W. Cohen, Technical Report WS-98-08, AAAI Press (1998) 714 .

[14] N. Good, J. B. Schafer, J. A. Konstan, A. L. Borchers, B. Sarwar, J. Herlocker, J. Riedl, Proc. Conf. Am. Assoc. Artif. Intell. (1999) 439.

[15] J.-G. Liu, M.Z.-Q. Chen, J. Chen, F. Deng, H.-T. Zhang, Z. Zhang, T. Zhou, Int. J. Inf. and Sys. Sci. 5(2) (2009) 230.

[16] J. B. Schafer, D. Frankowski, J. L. Herlocker, S. Sen, Lect. Notes Comput. Sci. 4321 (2007) 291.

[17] B. Sarwar, G. Karypis, J. A. Konstan, J. Reidl, Proc. ACM Conf. Electro. Commerce (2000) 158. 
[18] Y.-C. Zhang, M. Medo, J. Ren, T. Zhou, T. Li, F. Yang, Europhys. Lett. 80 (2008) 68003.

[19] T. Zhou, J. Ren, M. Medo, Y.-C. Zhang, Phys. Rev. E 76 (2007) 046115.

[20] Y.-C. Zhang, M. Blattner, Y.-K. Yu, Phys. Rev. Lett. 99 (2007) 154301.

[21] T. Zhou, L.-L. Jiang, R.-Q. Su and Y.-C. Zhang, Europhys. Lett. 81 (2008) 58004 .

[22] J.-G. Liu, Y.-Z. Dang, Z.-T. Wang, Physica A 366 (2006) 578.

[23] J.-G. Liu, Z.-G. Xuan, Y.-Z. Dang, Q. Guo, Z.-T. Wang, Physica A 377 (2007) 302.

[24] T. Zhou, L. Lü, Y.-C. Zhang, arXiv:0901.0553. 